\title{
AL CALOR DEL FUEGO: LA DIDÁCTICA MEDIANTE LA ARQUEOMETALURGIA EXPERIMENTAL Y EXPERIENCIAL
}

In the heat of fire: didactics through experimental and experiential archaeometallurgy

\author{
Beatriz Comendador Rey ${ }^{1}$, Aaron Lackinger ${ }^{1}$ y Elin Figueiredo ${ }^{2}$
}

https://doi.org/10.15366/baexuam2020.14.002

\section{RESUMEN}

En este artículo se sintetizan diversas acciones llevadas a cabo en el ámbito de la arqueometalurgia prehistórica y la arqueología experimental, con el fin de promover tanto su investigación académica, como su didáctica en distintos contextos educativos. En su mayor parte han estado dirigidos a que se conozcan los principales aspectos de la cadena técnica operativa teórica de producción del metal de base cobre y del hierro. En estas actividades, se busca aportar a los participantes las herramientas teóricas y empíricas básicas para la comprensión de los procesos metalúrgicos en la Prehistoria, a través del aprendizaje basado en la recreación tecnológica y al mismo tiempo, ofertar la participación activa en los procesos y su adquisición experiencial.

PALABRAS CLAVE: Arqueometalurgia, Experimental, Experiencial, Didáctica, Prehistoria

\begin{abstract}
In this paper, we resume several actions in the field of prehistoric Archeometallurgy and Experimental Archaeology. These actions has been carried out to promote both academic and didactic research, either in different educational contexts. Most actions, activities focused in the familiarization with the main aspects of the theoretical copper-based and iron production operative chains. These activities provided participants with basic theoretical and empirical tools for the understanding of metallurgical processes in prehistory. The learning through technological recreation provided an active participation in the processes, offering an experiential acquisition.
\end{abstract}

KEYWORDS: Archaeometallurgy, Experimental, Experiential, Didactics, Prehistory

\footnotetext{
${ }^{1}$ Grupo de Estudos de Arqueoloxía, Antigüidade e Territorio (GEAAT), Universidade de Vigo ORCID 0000-00027717-6464 beacomendador@uvigo.es

${ }^{2}$ Centro de Investigação em Materiais [CENIMAT/I3N], Faculdade de Ciências e Tecnologia, Universidade NOVA de Lisboa, Portugal ORCID 0000-0002-4821-3895 emf12055@ campus.fct.unl.pt
} 


\section{Introducción: presupuestos de partida}

En los últimos años hemos venido desarrollando toda una serie de actividades vinculadas con la arqueometalurgia prehistórica, bajo la etiqueta de arqueología experimental. Inicialmente, surgieron como un desarrollo lógico de la investigación más convencional sobre la actividad metalúrgica en la prehistoria, donde generalmente la teoría sobre los procesos tecnológicos dejaba abiertas más preguntas que respuestas. De ese modo, incorporaron el factor experiencial como vía exploratoria, hasta dar el salto a la experimentación. A continuación, nos proponemos realizar algunas reflexiones sobre el origen y desarrollo de estas actividades, sobre las premisas presentes y su potencial.

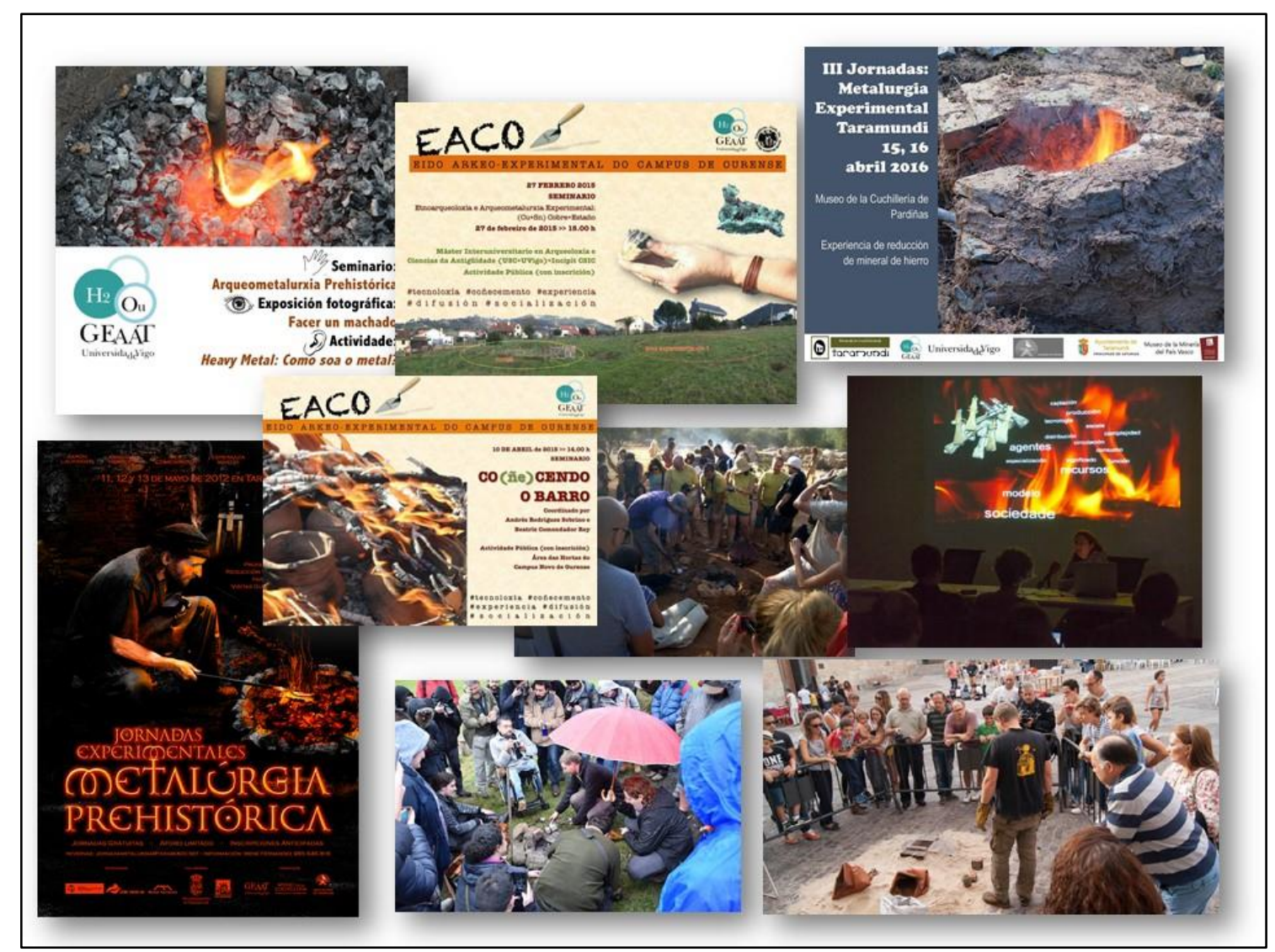

Figura 1. Diversos eventos organizados.

El desarrollo de la investigación se estructura alrededor del concepto de Cadena Técnica Operativa (CTO), definido por André Leroi-Gourham (Chaîne Opératoire) (1971) y que se emplea en arqueología para significar la secuencia de acciones implicadas en la producción de un artefacto. La antropología de la Técnica estudia los procesos implicados en las producciones humanas, siendo fundamental la diferencia entre técnica y tecnología, aunque ambas se relacionan, ya que van a determinar tanto el proceso como el resultado obtenido. Como ha señalado Marcos Martinón-Torres (2002) se trata más bien de un conjunto de enfoques complementarios que pretenden una comprensión holística del papel de las tecnologías en las sociedades pretéritas, y resulta una herramienta útil desde el punto de vista analítico. Podemos afrontar el estudio del registro arqueológico a través de la caracterización tipológica, pero la aproximación tecnológica acerca de los procesos 
implicados resulta ampliamente enriquecedora, estableciéndose el vínculo a través de la etnoarqueología.

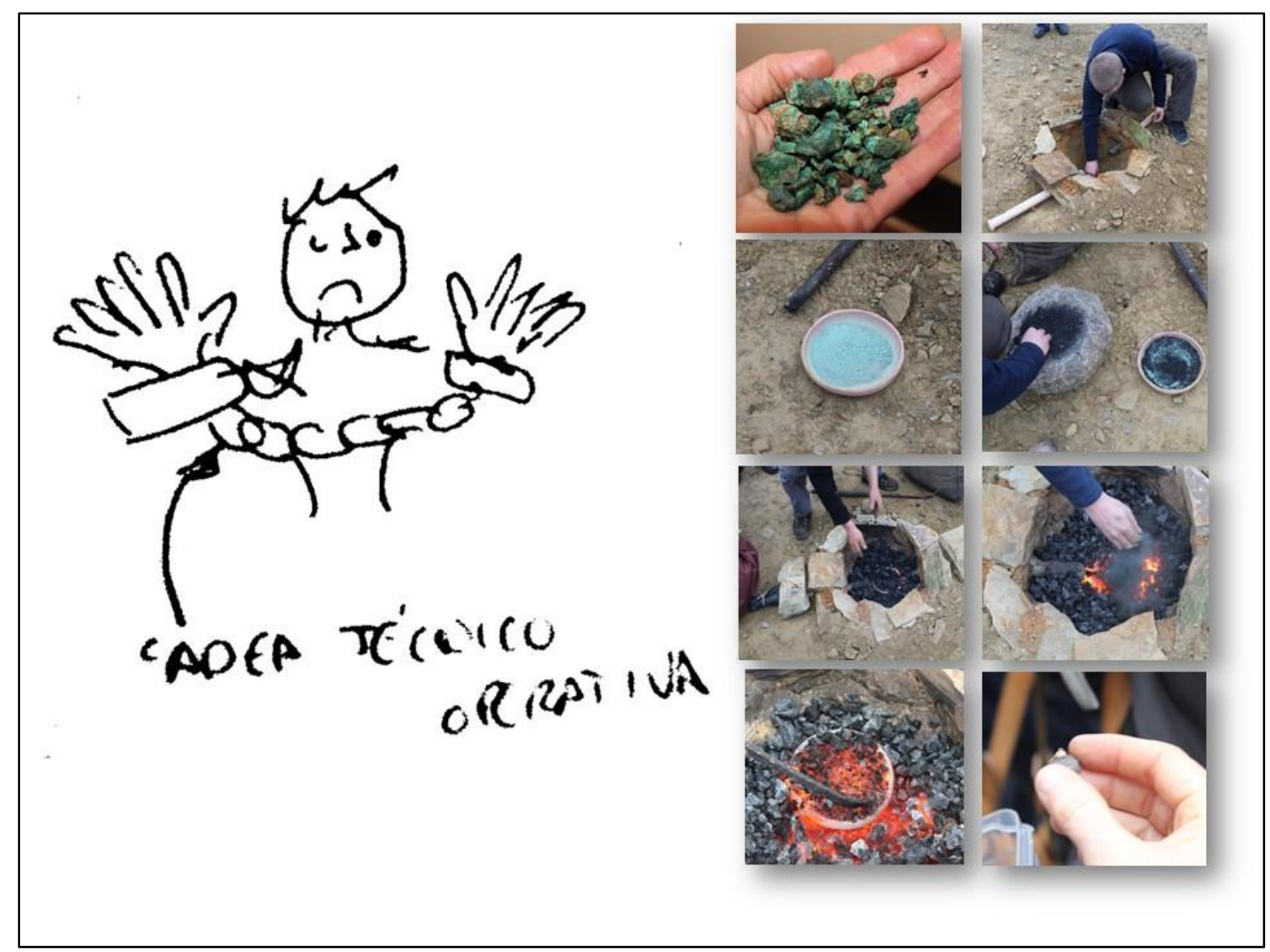

Figura 2. Dos visiones del concepto de Cadena Técnica Operativa (CTO): la visión limitante, en los materiales generados por los asistentes a los talleres didácticos de arqueometalurgia y la visión ordenada en una secuencia de acciones y elecciones desde la arqueometalurgia experimental.

El paso complementario es la experimentación en arqueología, una disciplina habitual en los estudios arqueológicos, especialmente desde los años 60 del pasado siglo XX, cuando comienza su desarrollo teórico (Ascher, 1963), siendo notable la pujanza de los estudios experimentales en toda Europa (Reeves y Paardekooper, 2014). En España, su emergencia reciente en el presente siglo es visible en el desarrollo de iniciativas como la publicación, desde 1997, del Boletín de Arqueología Experimental, o la celebración periódica desde el 2005, del Congreso Peninsular de Arqueología Experimental, vinculado a la propia creación un año antes, de Experimenta, la asociación española sobre esta materia (Ramos, González y Baena, 2006).

Esta creciente importancia se manifiesta también en su cada vez mayor presencia como disciplina en centros universitarios dedicados a la formación de arqueólogos y en su inclusión en los principales manuales de arqueología (Heeb y Ottaway, 2014).

La arqueometalurgia no es ajena a este impulso. Salvador Rovira ha expuesto la necesidad de realizar experimentación al respecto, "recreando los procesos teóricos sobre la metalurgia peninsular en las supuestas condiciones de trabajo del fundidor prehistórico", frente a estudios en condiciones de laboratorio (2007, p. 34). 
Las sesiones de trabajo propuestas para el congreso de arqueología experimental celebrado en Exeter en 2006 (Cunningham, Heen y Paardekooper, 2008) sintetizan las principales temáticas de interés al respecto:

1. Explorar el ámbito de la "arqueología experimental" y distinguir entre experimentos completos, experiencias-piloto, pre-experiencias, actividades experimentales, demostraciones públicas y proyectos de hobby.

2. El papel de la arqueología experimental en la investigación académica.

3. Mejora de los medios de promover la arqueología experimental en la investigación académica.

4. Mejorar la comunicación entre los arqueólogos experimentales académicos y artesanos.

5. Cómo comunicar experimentos para la comunidad académica y al público.

Siguiendo esta estructura, plantearemos los siguientes cinco apartados.

\section{La exploración en el ámbito de la "arqueología experimental"}

Tenemos que partir de la premisa de que no todo lo que se hace bajo la etiqueta de arqueología experimental lo es, o al menos, no se puede categorizar de igual manera. Javier Baena (1997, pp. 2-5) distingue entre: Modelos experimentales no rigurosos o de adquisición de pericia; Modelos rigurosos con poco o bajo control de variables; Modelos rigurosos con alto control de variables.

A pesar de que se suele denostar la vía experiencial, a favor de la experimental, nuestro punto de partida fueron diversas experiencias piloto o pre-experiencias, como vía exploratoria, para la adquisición de destrezas o prueba de equipamientos. La experimentación también tiene un papel heurístico, aunque el término experimento debe ser utilizado solo para aquellos que adoptan claramente una aproximación científica (Morgado y Baena, 2011, pp. 21-28; Baena et al, 2019). Como se ha señalado, siempre hay, por supuesto, un elemento de experiencia en un experimento, pero no siempre un experimento en una experiencia (Cunningham, Heen y Paardekooper, 2008, pp. 71-75).

Desde un principio tendimos a realizar jornadas experimentales, concebidas como demostraciones públicas con fines didácticos orientadas a mostrar distintos procesos relacionados con las tecnologías prehistóricas, y asumiendo la premisa de la capacidad didáctica de los errores (Flutsch, 1994).

\section{Arqueología Experimental e investigación académica}

El papel de la arqueología experimental en la investigación académica es poner en práctica modelos propuestos de manera teórica, con el objeto de validar o modificar las premisas testadas, así como explorar y obtener nuevas ideas; al mismo tiempo aportan 
importante información de carácter sensorial y técnico a las investigaciones arqueológicas.

En la arqueometalurgia, las lecturas en torno a la fabricación de objetos arqueológicos son de gran dificultad, ya que, de forma general, el objeto arqueológico metálico se ha producido a través de una sucesión de procesos independientes, cuyo desarrollo no es rastreable partiendo únicamente de un artefacto. Como modelo general teórico, adoptamos para su estudio un enfoque biográfico que presupone una fase de producción, una de circulación y otra de amortización o consumo. En cuanto a la producción en concreto, se distingue en primer lugar la fase de Metalurgia Extractiva, que engloba el abastecimiento de los materiales necesarios para la producción metalúrgica posterior, como el proceso metalúrgico, propiamente dicho, consistente en la transformación físicoquímica o reducción de minerales a través de un proceso pirometalúrgico en metales o aleaciones. En segundo lugar, estaría la fase de elaboración de objetos a partir del metal obtenido, que comprende procesos diversos, de complejidad variable, en líneas generales podrían resumirse en fundición (puede incluir un proceso de aleación, o mezcla intencional de distintos metales), colado en molde, deformación plástica (forja, laminado, trefilado, estampado... que pueden requerir procesos de térmicos), ensamblado (perforación, remachado, soldadura, pegado, atado... puede implicar materiales metálicos y no metálicos), acabado (pulido, afilado, decoración...), reparación (reafilado, soldadura, sobrefundido, perforación, remachado...), reutilización (con una función diferente de la original, de parte o del total de un objeto), reciclaje y amortización. Todas o alguna parte de esta secuencia se encuentran presentes en los objetos metálicos arqueológicos.

Para obtener información acerca de la CTO de un artefacto, resulta fundamental el contexto arqueológico y las evidencias detectadas sobre el proceso productivo. A través de las variadas técnicas disponibles hoy en día y de los conocimientos adquiridos en el área de la ciencia de los materiales (estudios arqueometalúrgicos), se puede inferir alguna información de la CTO, aunque no es posible hacer una lectura secuencial completa. Por ejemplo, a partir de los análisis de caracterización elemental, se puede establecer la ley del metal o aleación empleada; a partir de los análisis metalográficos se puede observar la microestructura de los objetos, obteniéndose información acerca de los procesos finales de obtención de forma de los objetos; a través de los análisis de isótopos se podrá obtener indicaciones sobre la discriminación de las procedencias del metal en relación con los depósitos minerales. Sin embargo no es posible determinar con certeza como era obtenida la aleación: ¿adición de diferentes metales o reducción simultánea de diferentes minerales? No es posible saber la eficacia real de uso de un objeto así como su tiempo de "vida", y en ocasiones puede resultar difícil discriminar si serían mezclados minerales de diferentes orígenes, si los metales serían frecuentemente reciclados o no, y cual la ventaja de reciclar versus reparar una pieza partida o defectuosa.

A partir de esta información y de la etnoarqueología, se proponen modelos teóricos de producción metalúrgica, teniendo en cuenta las evidencias en contexto arqueológico. Estos modelos constituyen la base para el diseño de los experimentos arqueometalúrgicos que realizamos. Partiendo de estas premisas, hemos promovido el desarrollo de estas investigaciones ya en relación con la actividad docente universitaria y especialmente en 
el marco de trabajos académicamente dirigidos ${ }^{3}$, ya en el desarrollo de una red de investigación.

Así, en 2010 creamos el grupo de trabajo Early Tin Iberian Group a partir de sinergias entre investigadores de diferentes instituciones en España, Portugal y Francia ${ }^{4}$. Actualmente, derivado de aquel equipo, se desarrolla un proyecto internacional sobre el estaño ${ }^{5}$. Recientemente, el componente experimental se incorporó también en el marco del proyecto estatal Producción y deposición masiva de bronces plomados en la transición Bronce Final-Edad del Hierro de la Europa atlántica ${ }^{6}$.

En relación con la fase de metalurgia extractiva, no hemos desarrollado propuestas experimentales relacionadas con la forma de obtención de los minerales metálicos, si bien desde un principio hemos procurado introducir minerales que nosotros mismos hemos obtenido en el noroeste peninsular, o directamente de escombreras de minas históricas o a través de la colaboración con aficionados a la mineralogía ${ }^{7}$ o geólogos ${ }^{8}$. En el caso del cobre, hemos venido trabajando con malaquita de la zona de Valdeorras (Lackinger et al., 2013), y en el caso del estaño, hemos trabajado con casiterita de las minas de Penouta (Viana do Bolo, Ourense) y Gondiães (Portugal).

Del mismo modo, en el marco del Early Iberian Tin Group, hemos presentado la vía experimental como un elemento fundamental de investigación (Comendador et al., 2015),

\footnotetext{
${ }^{3}$ Las primeras actividades tuvieron lugar en el marco de la investigación académica dirigida de A. Lackinger (2008, 2011 y 2016) con trabajos de investigación desarrollados en la Universidad de Vigo y la Universidad de Granada, y en colaboración con la beca posdoctoral de E. Figueiredo financiada por la Fundaçâo para a Ciencia e a Tecnología y co-orientada por la Universidade Nova de Lisboa, Universidade de Évora y la Universidade de Vigo.
}

${ }^{4}$ Integrado por investigadores de la Universidad de Vigo; Laboratoire TRACES (CNRS), University of Toulouse, Francia; Centro de Investigação em Materiais (CENIMAT/I3N), Faculdade de Ciências e Tecnologia, Universidade NOVA de Lisboa, Portugal; Instituto de Ciencias del Patrimonio INICIP_CSIC, España; Faculdade de Ciências, Universidade do Porto, Portugal; Laboratório HERCULES, Universidade de Évora, Portugal.

${ }^{5}$ PTDC/HAR-ARQ/32290/2017 IberianTin - Ancient production, uses and circulation of tin in the NW Iberia. El proyecto está liderado por Elin Figueiredo, de la Universidade Nova de Lisboa.

${ }^{6}$ ATLANTAXES, HAR2017-84142-R liderado por el INCIPIT-CSIC con financiamiento del Ministerio de Economía, Industria e Competitividad a través da convocatoria de Proyectos Retos. El investigador principal es el Dr. Xosé Lois Armada, investigador Ramón y Cajal del INCIPIT-CSIC.

7 Queremos agradecer especialmente su inestimable ayuda a José Fernández, "Pepe do Cañete" de Vilamartín de Valdeorras, así como al arqueólogo Santiago Ferrer.

${ }^{8}$ Queremos agradecer especialmente la colaboración de Alexandre Lima, geólogo y profesor de la Faculdade de Ciências de la Universidade do Porto. 
lo que nos posibilitó, ya desenvolver investigación científica en el ámbito de la reducción ya de estaño solo, ya conjuntamente con cobre.

En cuanto a los experimentos relacionados con la reducción de mineral de base cobre, a nivel teórico, se ha propuesto que existen tres formas posibles de fabricación de bronce, certificándose que todas ellas fueron empleadas en la Península Ibérica entre la Prehistoria y la Protohistoria (Rovira, 2007, pp. 21-22). La primera de ellas, denominada co-reducción, consiste en la obtención de bronce a partir de una mezcla de minerales de cobre y estaño, que se procesan conjuntamente en una estructura metalúrgica. La segunda conocida como cementación consiste en la obtención de la aleación mediante la reducción de minerales de estaño sobre cobre metálico. La última de las fórmulas posibles de obtención de bronce es la reducción por separado de minerales de cobre y estaño, para su posterior aleación Las actividades que hemos desarrollado han explorado de forma experimental estas tres posibilidades de forma positiva, permitiéndonos profundizar en la metalurgia extractiva desde una perspectiva empírica (Lackinger et al., 2013; Figueiredo et al., 2017).

En cuanto a la elaboración de artefactos de tipologías prehistóricas, hemos explorado distintos materiales y técnicas. Así se ha testado el uso de moldes cerámicos, tanto bivalvos como a la "cera perdida" (Lackinger y Comendador, 2013), moldes bivalvos de piedra, tanto arenisca como esteatita (Lackinger, 2014). Estos experimentos nos ayudan a mejorar nuestra comprensión en torno a los materiales y técnicas empleadas durante la Prehistoria para la fabricación de objetos de bronce.

Hasta el momento no hemos desarrollado experimentación en cuanto a la utilización de artefactos, si bien uno de nosotros realizó un estudio sobre las formas de enmangue de las hachas planas de cobre (Comendador y Méndez, 2007).

Más recientemente hemos desarrollado las primeras experiencias relacionadas con la reducción de hierro, aunque se trata aún de un acercamiento preliminar, con resultados modestos, que aún están en proceso de valoración y que esperamos ampliar en el futuro.

En las experiencias de naturaleza experimento, se controlan cantidades de minerales usadas, tiempos y temperaturas. As temperaturas se controlan a través de un termopar tipo K, acoplado a un equipo TC-08 Pico, monitorizado por un ordenador portátil, empleando el software propio del equipo. Fundamental para la labor desarrollada, es el apoyo logístico de un equipo de investigadores, especialistas en arqueometalurgia, lo que nos permite implementar resultados analíticos posteriores a nuestras investigaciones experimentales. Para los análisis de los materiales implicados en el proceso de reducción, tanto los previos (carbón, minerales y cerámica del crisol) como los posteriores (estaño, cobre, bronce, escorias y cerámica de crisol), tras su cuantificación, se toman muestras representativas de cada uno de los productos. Los análisis efectuados han sido de naturaleza elementar (composición), mineralógica y microestructural. Las técnicas usadas con mayor frecuencia son las de la Espectrometría de Fluorescencia de Rayos X (XRF), Difracción de Rayos X (XRD), Microscopía Óptica (OM) y Microscopía Electrónica de Barrido (SEM-EDS). También han sido realizados análisis isotópicos de estaño en el ámbito de una colaboración internacional con el proyecto proyecto Bronze Age tin - Tin 
isotopes and the sources of Bronze Age tin in the old World (ERC project). Los resultados de estas investigaciones están siendo presentados en diversos foros nacionales e internacionales (Berger et al., 2018; Figueiredo et al., 2017).

Resulta importante señalar, que muchas de estas actividades se han venido realizando, hasta el momento en la Península Ibérica, sin financiación obtenida a través de un proyecto específico de convocatoria pública, o tan siquiera un apoyo financiero estable. Son por tanto fundamentalmente las sinergias de trabajo, la colaboración entre investigadores e instituciones, y la voluntad decidida a abrir esta vía de investigación, las que soportan las actividades que acabamos de describir.

\section{Arqueología Experimental en el sistema universitario gallego}

La arqueología experimental como vía didáctica en la formación reglada de arqueólogos está presente en los planes de estudio de distintas universidades. En el contexto británico, el desarrollo de la arqueología experimental en el ámbito académico tiene una larga trayectoria, e incluso hay masters específicos al respecto, como el de la Universidad de Exeter $^{9}$ o en el UC de Dublín ${ }^{10}$. En ambos se focaliza el estudio en los principios de la arqueología experimental y en las herramientas para la investigación de materiales arqueológicos, pero también en la gestión y comunicación del patrimonio cultural, dado su importante papel como herramienta en el análisis y valorización del mismo.

En el ámbito de las universidades españolas, y concretamente en el de la arqueometalurgia experimental, destacan los experimentos iniciados por Salvador Rovira en la Universidad Autónoma de Madrid (2011-12) y que tienen continuidad en el actual Laboratorio de Arqueología Experimental-UAM; así mismo la Universidad de Granada, en su Máster de Arqueología, cuenta específicamente con un taller de minería y metalurgia, en que se incluye una actividad denominada: "La experimentación y recreación de los procesos metalúrgicos del Cobre".

En el mapa de titulaciones del sistema universitario gallego, donde no hay un grado específico en Arqueología, y las titulaciones vinculadas son de ámbito generalista, la arqueología experimental no ha tenido una presencia formal expresa, si bien se venían desarrollando diversas actividades buscando las sinergias al respecto, tanto desde el Grupo de Estudios para la Prehistoria del Noroeste Ibérico (GPEN) de la USC, como desde el Grupo de Estudios de Arqueología, Antigüiedad y Territorio (GEAAT) de la Universidad de Vigo.

Por parte del GEAAT, las primeras "Jornadas de Metalurgia Prehistórica Experimental" se organizaron en Mayo de 2012 en Taramundi, en colaboración con Ceder Oscos-Eo, el Ayuntamiento y el "Museo de la Cuchillería" de Taramundi, así como la Asociación de

${ }^{9}$ http://www.exeter.ac.uk/postgraduate/taught/archaeology/experimentma/

${ }^{10}$ http://www.ucd.ie/archaeology/study/graduateprogrammes/msc experimental/ 
Herreros "Consejo del Hierro". Estuvieron fundamentalmente orientadas a la experimentación en la producción de bronce. A partir de las "II Jornadas de Metalurgia Prehistórica Experimental" "11 celebradas en diciembre de 2014, éstas se orientaron a la producción de hierro. Las III Jornadas se celebraron en abril de $2016^{12}$ y las IV en diciembre de 2018, siempre en la localidad asturiana de Taramundi, bajo el auspicio de su Excmo. Ayuntamiento y del "Museo de la Cuchillería".

Además los integrantes del GEAAT participaron en diversas jornadas abiertas, como las "I Jornada de Recreació Prehistòrica Jaciment Arqueològic dels Closos de Can Gaià" (Portocolom, Felanixt, Mallorca, Agosto 2013), en la "Jornada Prehistórica de Arqueología Experimental en Fuentes de León” (Badajoz, Diciembre 2013), así como la colaboración con el Parque Arqueológico de Arte Rupestre de Campo Lameiro (PAAR) en la organización del curso "Transformando materiales, una aproximación interdisciplinar a la producción de objetos de bronce" (Octubre 2013).

Desde el inicio de estas actividades, resultó claro el interés tanto del alumnado universitario como del público en general, con una participación asidua y reiterada de asistentes. Sobre la base de estas experiencias anteriores, la comisión que diseñó el Máster interuniversitario en Arqueología y Ciencias de la Antigüedad (USC, Uvigo, INCIPIT-CSIC) en el curso 2013-14, promovió la creación de la materia optativa "Etnoarqueología y Arqueología Experimental". Desde su implantación en el curso 2014-15, las actividades se desarrollan en relación con este contexto docente formal, así como promoviendo la realización de trabajos académicos.

Así en el curso 2014-15, se celebraron tres jornadas experimentales; una en el espacio experimental del Monte da Condesa del Campus Sur de la Universidad de Santiago de Compostela y a través del GEPN ${ }^{13}$, y dos en el Campus de Ourense de la Universidad de Vigo a través del GEAAT, con el apoyo de la Vicerrectoría (Figs. 3 y 4). Los buenos resultados obtenidos en este curso promovieron incluso la propuesta de creación de un espacio arqueo-experimental en el campus de Ourense (EACO) como un recurso educativo.

\footnotetext{
${ }^{11}$ Las II Jornadas de Metalurgia Prehistórica Experimental se realizaron en con la colaboración de D. Luis Padura Elorza, maestro herrero, hoy jubilado, con amplia experiencia en la obtención de hierro a partir de sus minerales, parte de la cual se vincula a las actividades desarrolladas en la Ferrería del Pobal, en Muskiz (Vizcaya).

${ }^{12}$ En esta ocasión contamos con la colaboración de Museo de Minería del País Vasco, de la mano del arqueólogo Javier Franco, actualmente secretario de la Asociación Cultural Museo Minero.

${ }^{13}$ http://gepn.jimdo.com/galería/
} 


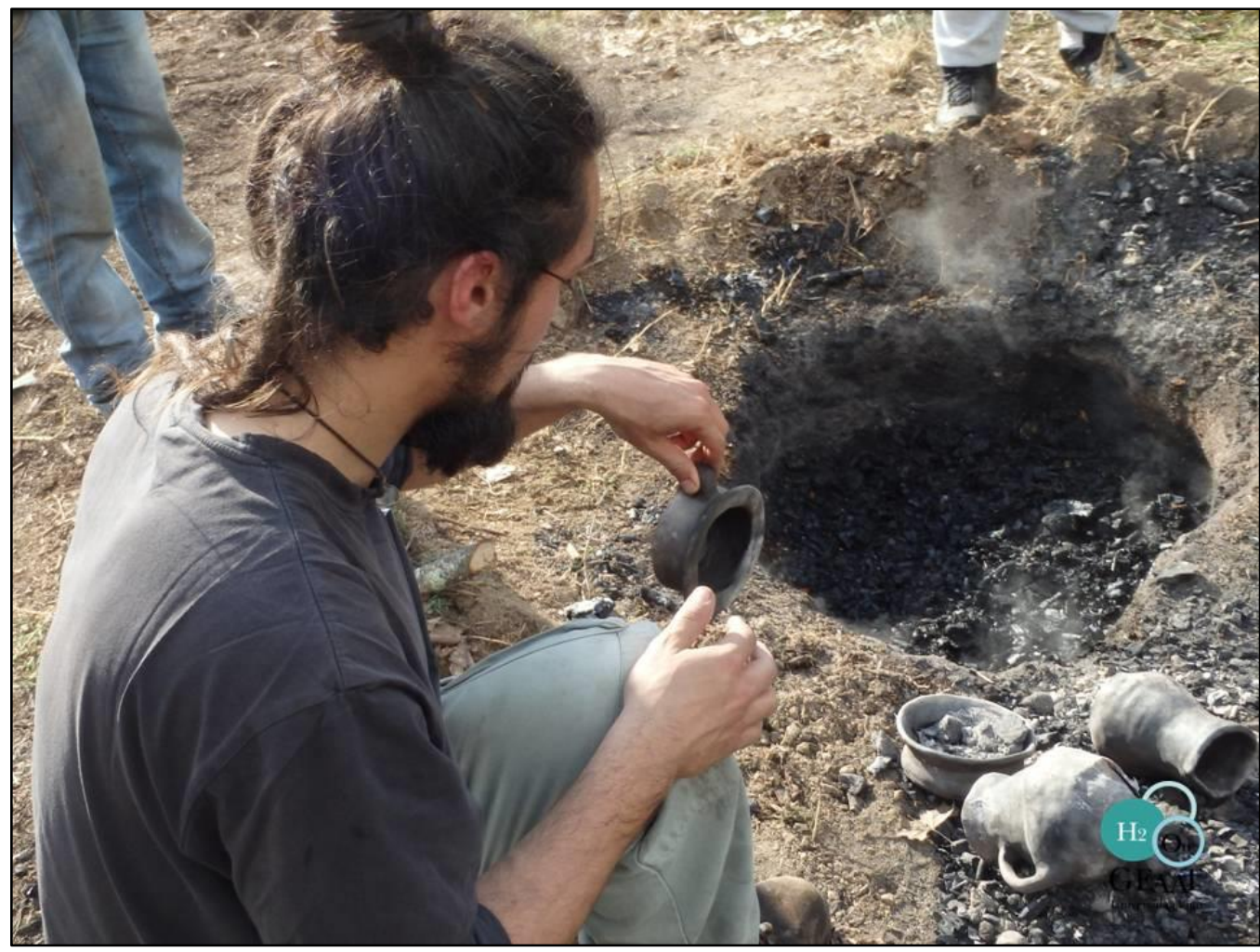

Figura

3. Jornada de cocción de cerámica en el Espacio Experimental del Campus de Ourense EACO de la Universidad de Vigo (2015)

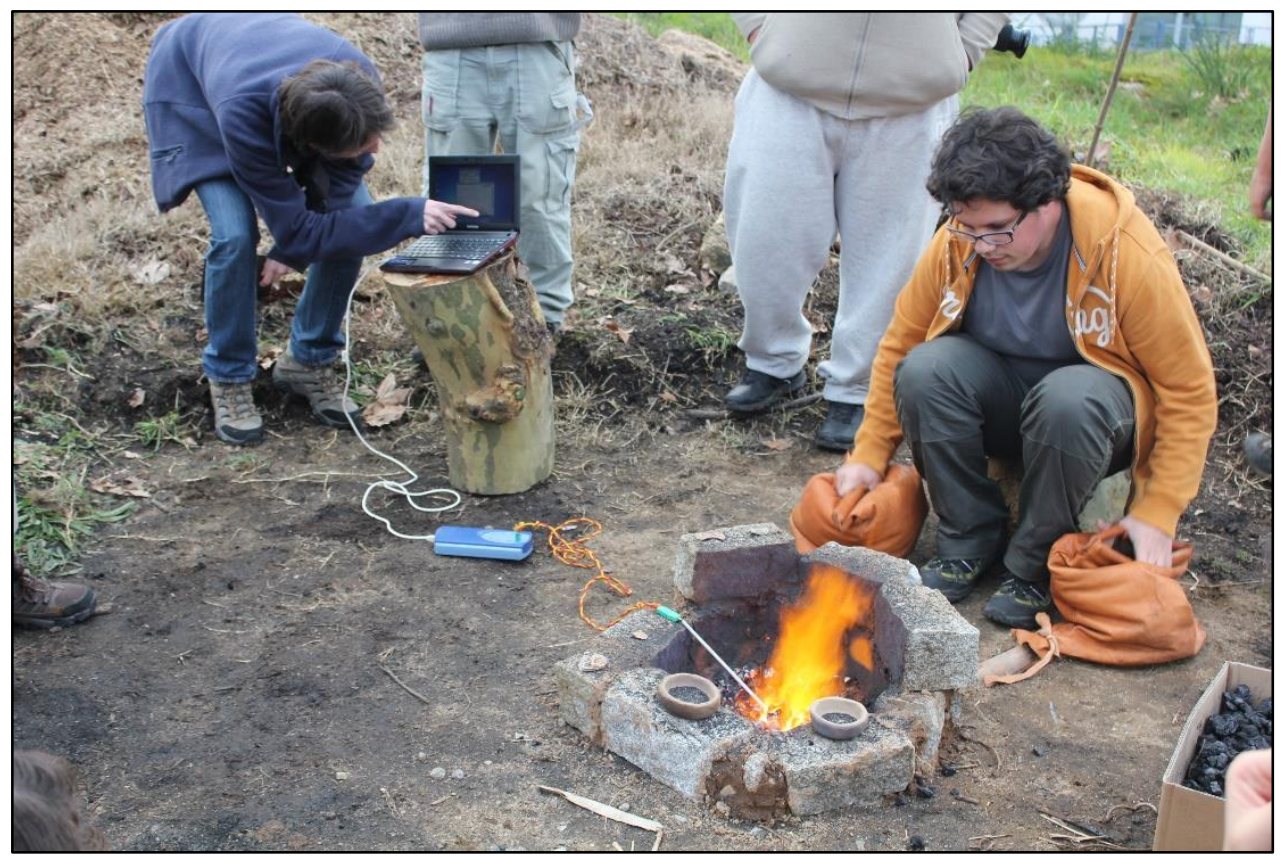

Figura 4. Jornada de arqueometalurgia en el Espacio Experimental del Campus de Ourense EACO de la Universidad de Vigo (2015)

En el curso 2015-16, las actividades se realizaron a través del GEAAT, en colaboración con el PAAR de Campo Lameiro. Se organizaron como curso, con amplio éxito de asistencia (Fig. 5), mostrando el potencial de este tipo de eventos. Así mismo se potenció la participación del alumnado en las "II Jornadas de Metalurgia Prehistórica Experimental" celebradas en Taramundi, anteriormente citadas. En el curso 2016-17, 
también en colaboración con el PAAR de Campo Lameiro, se organizó el evento Una Giornata al Sole $^{14}$ (Fig. 6). En los cursos 2017-18 y 2018-19, las actividades de la materia Etnoarqueología y Arqueología Experimental se desarrollaron en el espacio experimental del Monte da Condesa del Campus Sur de la Universidad de Santiago de Compostela (Fig. 7).

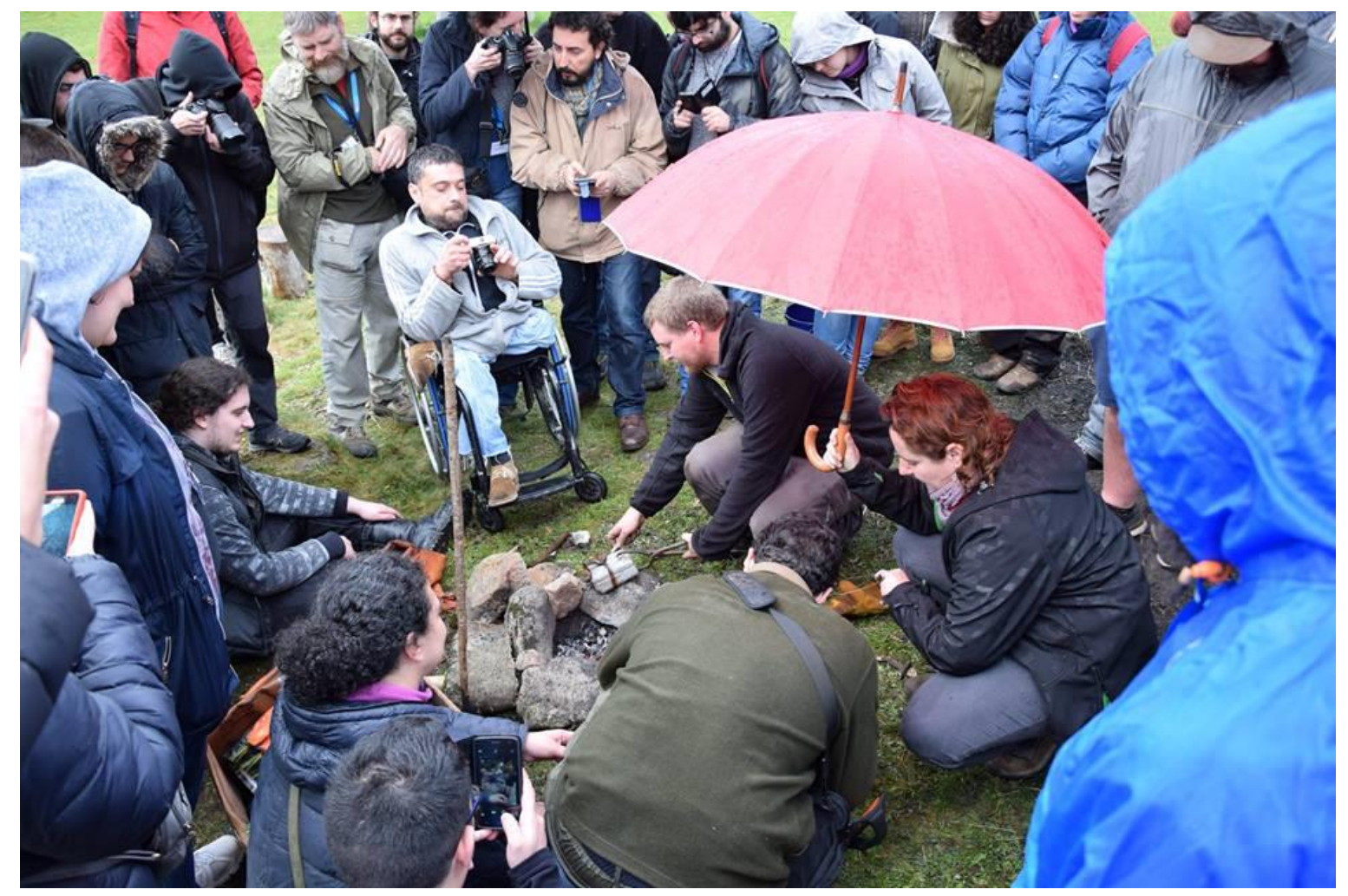

Figura 5. Evento experimental organizado por el GEAAT, en colaboración con el PAAR de Campo Lameiro (Campo Lameiro, 2016)

\footnotetext{
${ }^{14}$ Vídeo: https://www.facebook.com/watch/?v=1336992283051071
} 


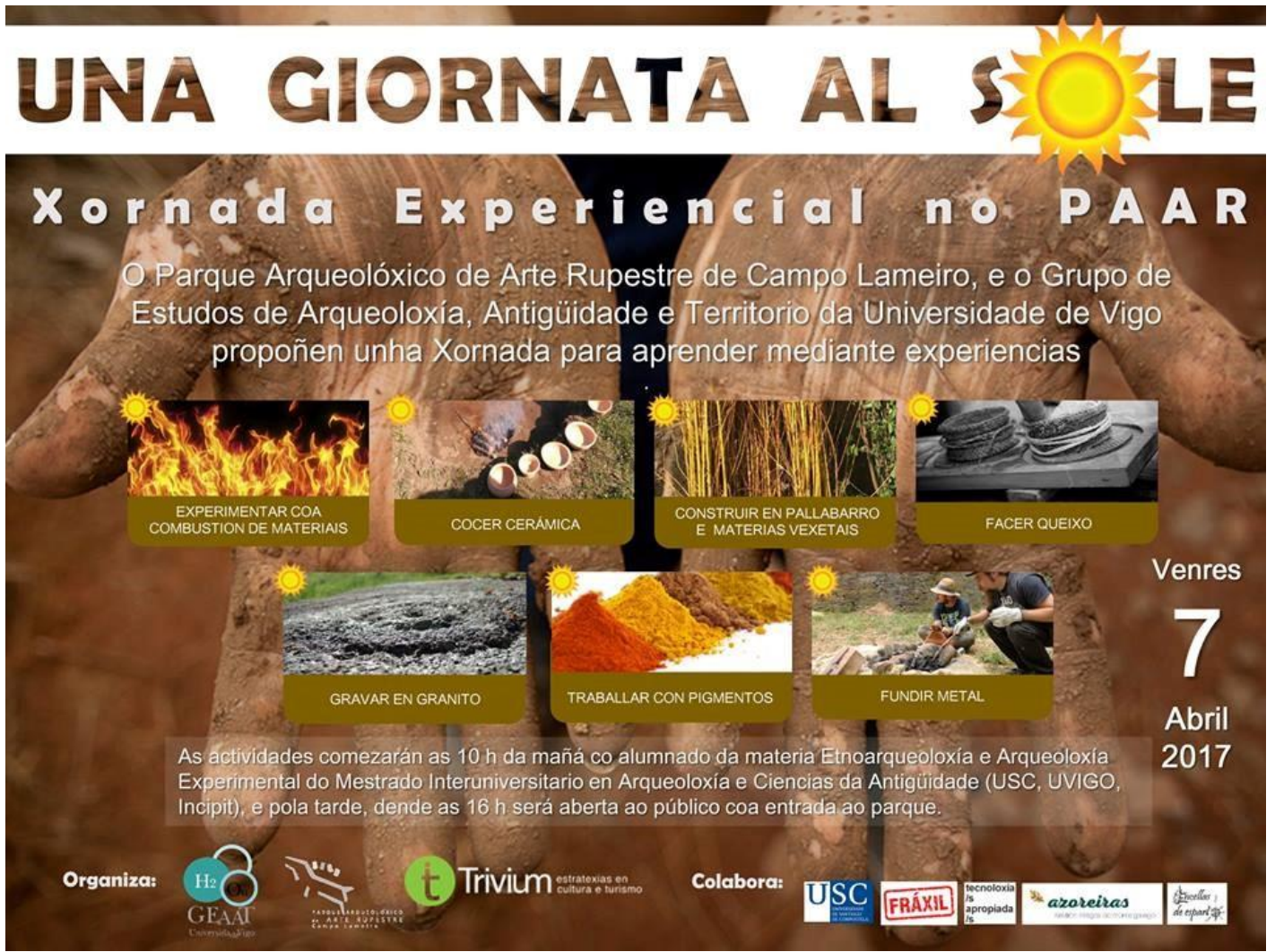

Figura 6. Evento Una Giornata al sole, en el PAAR de Campo Lameiro (Pontevedra, 2017)

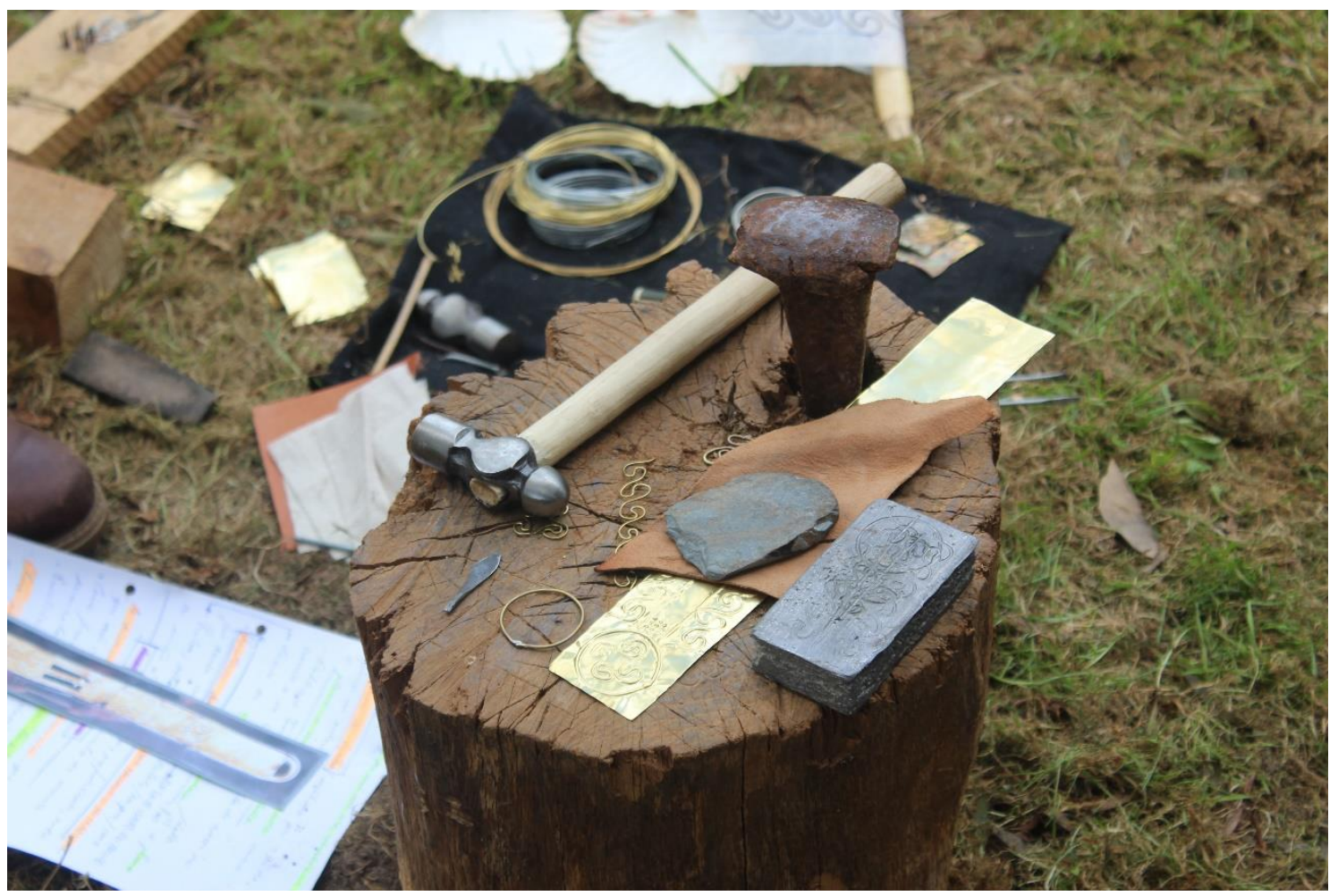

Figura 7. Jornada Experimental en el espacio experimental del Monte da Condesa del Campus Sur de la USC (Santiago de Compostela, 2018)

Un buen reflejo de la repercusión de estas actividades, es el dinamismo de las jornadas organizadas por estudiantes en el ámbito universitario. Así la "Asociación Cultural de 
Arqueoloxía e Ciencias da Antigüidade" (ARCIAN) ${ }^{15}$, organizó en 2016 una jornada experimental con demostraciones públicas en el campus de la USC. Por su parte, las Jornadas de Jóvenes investigadores organizadas por la Delegación de Alumnos de la Facultad de Historia del Campus de Ourense de la Universidad de Vigo, también vienen incluyendo en sus programas, demostraciones públicas y actividades de reenactment.

Por otro lado, algunos centros de educación primaria y secundaria han comenzado a demandar este tipo de actividades, dado su alto potencial didáctico transversal. Así por ejemplo, el GEAAT y el GEPN participaron conjuntamente con en la "jornada de experimentación" 16 organizada a solicitud del IES Marco do Camballón de Vila de Cruces (Pontevedra).

El balance es muy satisfactorio, puesto que nos muestra tanto el interés del alumnado, como la promoción de nuevos grupos de experimentación, como "Encellas de Esparto"17, pero todavía queda mucho camino por recorrer en la formalización de esta disciplina, así como en la creación de la sinergia institucional precisa para dar el apoyo a este tipo de formación.

Finalmente, en el año 2019, el GEAAT de la Universidad de Vigo entró formalmente a formar parte como miembro del EXARC ${ }^{18}$.

\section{Diálogos entre disciplinas}

La práctica de actividades "al calor del fuego" que realizamos en la vida cotidiana contemporánea, se traduce casi exclusivamente a la cocina, a pesar de que ya no se "encienden muchos fogones". La sociedad de consumo mantiene en la distancia los procesos tecnológicos, hasta tal punto, que resulta exitoso y de interés el formato de programas televisivos en los que se muestra "el proceso complejo que hay detrás de cada objeto cotidiano". El momento histórico actual, descrito por algunos como la era tecnológica, es, gran medida, ajeno a las implicaciones tecnológicas de los productos consumidos, lo que se debe a la desaparición de los modos de vida tradicionales, donde las actividades artesanales, hacían patente este nexo (Lackinger, 2016). A día de hoy la separación de la producción en áreas de máxima especialización, impiden al individuo asimilar el proceso que se esconde detrás de cualquier artefacto, por eso, la recreación de los mismos, como por ejemplo una posible CTO para la obtención de metal a partir de

\footnotetext{
${ }^{15}$ Fundada en 2014 por un grupo de estudiantes de la Universidad de Santiago de Compostela (USC) con el objetivo de estimular la investigación en las especialidades de Arqueología, Prehistoria e Historia Antigua entre los jóvenes investigadores.
}

${ }^{16}$ http://www.farodevigo.es/portada-deza-tabeiros-montes/2015/05/13/camballonprehistoria/1238907.html

${ }^{17}$ https://encellasdeesparto.wordpress.com/

18 https://exarc.net/members/high-ed/geaat-es 
mineral, o la producción de un artefacto metálico, causan un gran impacto y generan interés (Fig. 8).

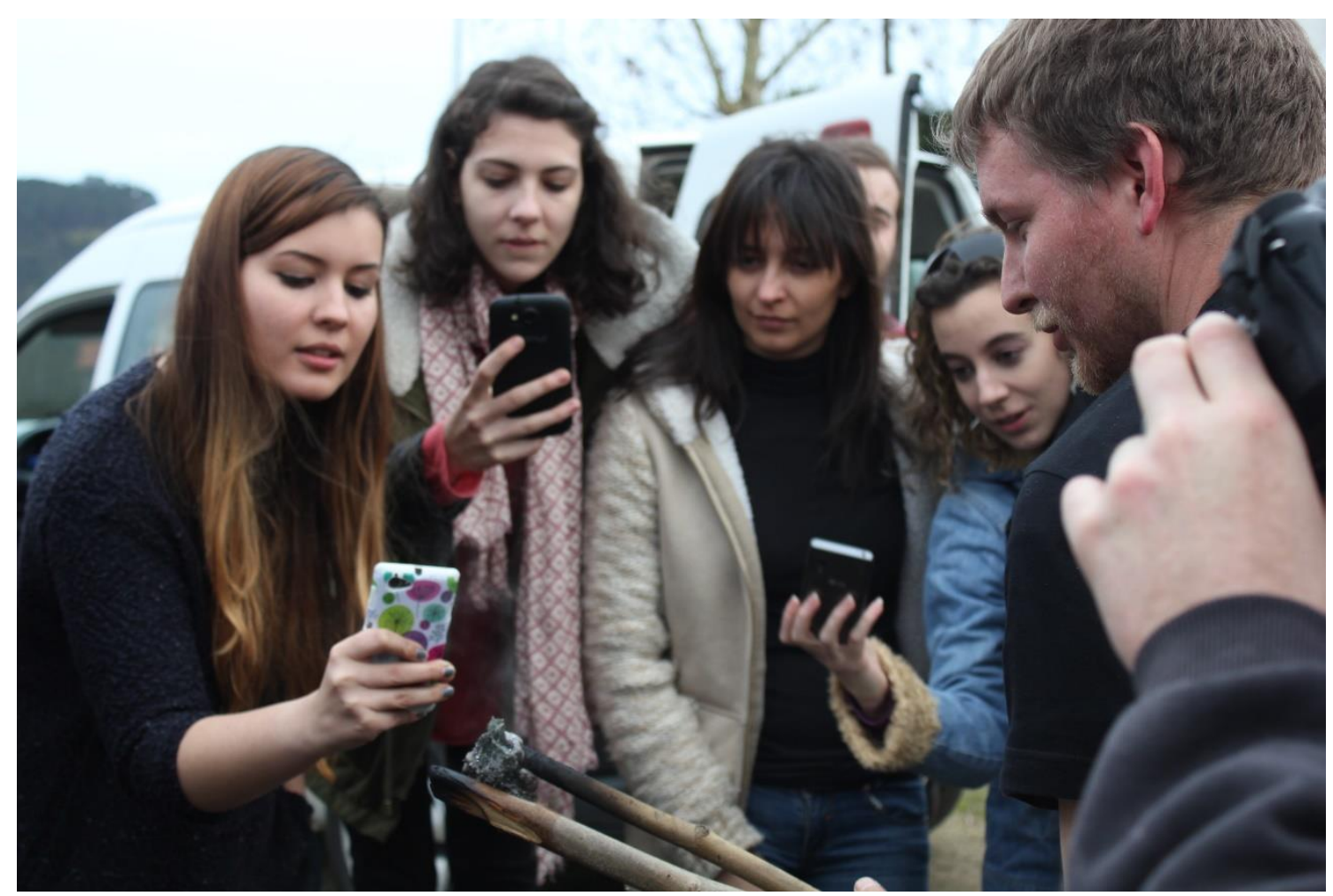

Figura 8. Expectación paleotecnológica en las jornadas realizadas en el Campus de Ourense de la Universidade de Vigo (Ourense, 2014)

Al mismo tiempo, pone de manifiesto la necesidad de promover el conocimiento de las actividades artesanales y el intercambio de experiencias, ya que supone un diálogo entre disciplinas ampliamente enriquecedor. El trasvase de ideas acerca de los procesos experimentados a pie de la hoguera, en diversos eventos, generó no solo interesantes conversaciones, sino también el entendimiento entre lenguajes de distintos ámbitos: el arqueológico, más apegado al registro y a las evidencias arqueológicas; el físico-químico y arqueométrico, más apegado a la ciencia de los materiales; y finalmente el artesanal, profundamente en contacto humano con la parte material y experiencial, cuya perspectiva resulta imprescindible. Valga como ejemplo la investigación etnoarqueológica realizada en cuanto a la obtención tradicional de estaño en Galicia (Fig. 9) (Lackinger et al., 2017). 


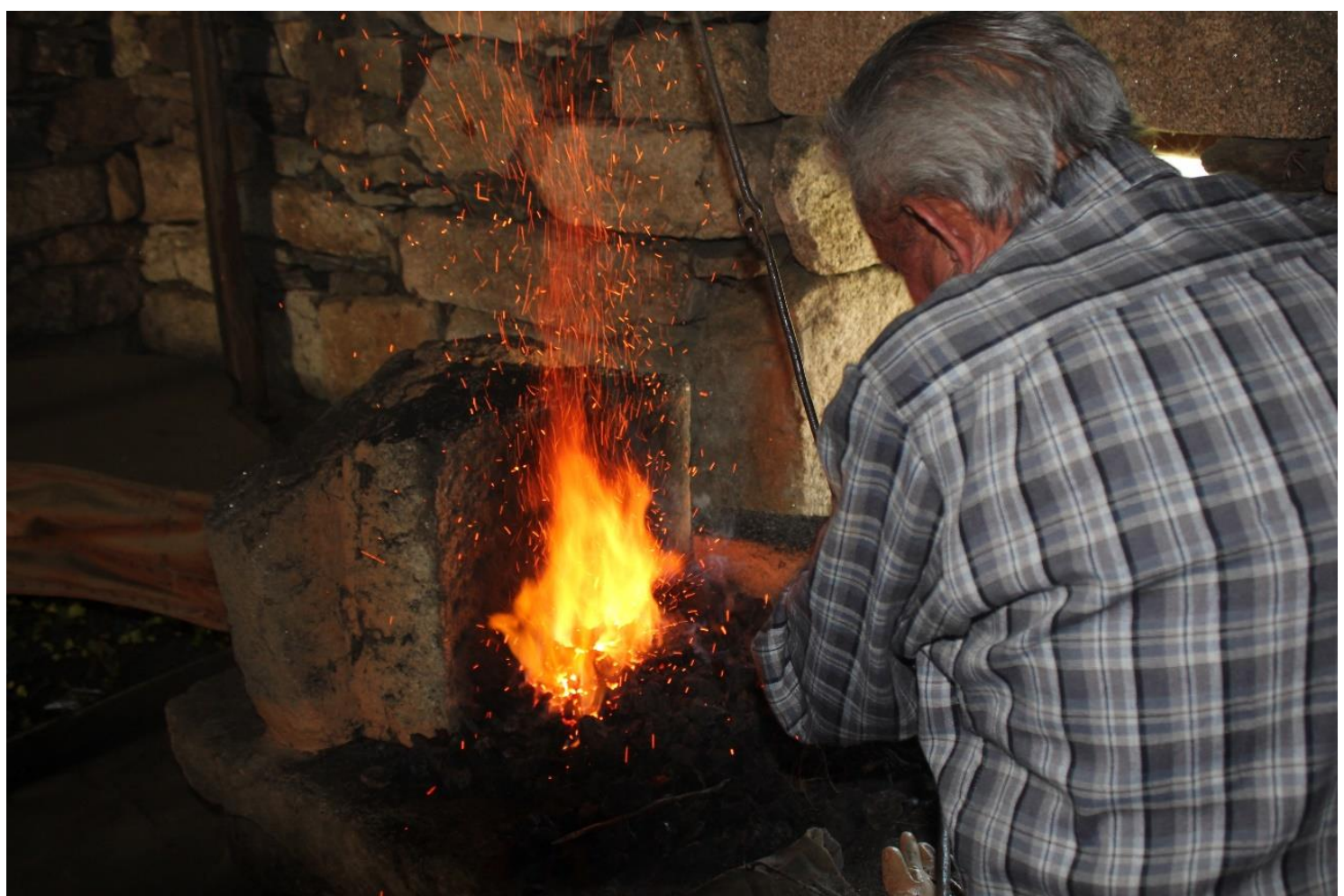

Figura 9. Documentación de un procedimiento tradicional de reducción de estaño en Pentes, A Gudiña (Ourense, 2013)

Por esta razón entre otras, resulta fundamental la antropología para la interpretación de los restos arqueológicos en su dimensión cultural más amplia y las posibilidades de la arqueología experimental en el estudio arqueométrico de los procesos de comportamiento de las tecnologías del pasado, pero también en la didáctica y sociabilización del conocimiento generado por la investigación arqueológica.

Finalmente, sostenemos que otro objetivo es concienciar de la necesaria relación transdisciplinar y dialógica que la arqueología experimental mantiene con la difusión del conocimiento arqueológico y la creación de una conciencia crítica sobre el valor del patrimonio cultural y su uso social.

\section{Comunicar y transferir}

En los últimos años, la Arqueología y el ámbito del Patrimonio Cultural, han reflexionado sobre su repercusión social como pocas disciplinas lo han hecho. Y esto tiene que ver con la difusión, no solo entre grupos más restrictos y especializados (p. ej. académicos), sino a toda una comunidad o público en general. Los nuevos paradigmas de la profesión hacen inexcusable no atender a este tipo de tareas a través de revistas de tipo divulgativo, prensa, y otra serie de eventos dirigidos a la sociedad en su conjunto. Sin embargo esta tarea carece de reconocimiento en el ámbito académico, donde se considera una actividad secundaria o poco valorada fuera del "núcleo duro". Por otro lado, el paradigma jerárquico, basado en el modelo de primero investigar y posteriormente divulgar los resultados, sigue siendo el predominante, aunque las cosas están empezando a cambiar por los crecientes procesos de empoderamiento de la sociedad civil sobre el patrimonio 
arqueológico y cultural en general, así como la toma de protagonismo en las tareas de gobernanza.

En los últimos años, se han multiplicado los eventos de recreación (living history), aunque algunos con muy escasa capacidad didáctica, en los que el principal ingrediente es el atrezo y que no constituyen más que actividades abiertas bajo la etiqueta de arqueología experimental, pero que no lo son. Se trata de una simple transmutación a la arqueología social/didáctica de algo que no lo es, y que una vez más disfraza de didáctico, lo que apenas se queda en lúdico con ambientación histórica (Morgado y Baena, 2011, pp. 2425). Su práctica supone una forma de teatro moderno, aun sin despreciar el rol del teatro en cuanto que es en la mayor parte de las veces es la forma más significativa en que nuestra disciplina interactúa con la gente.

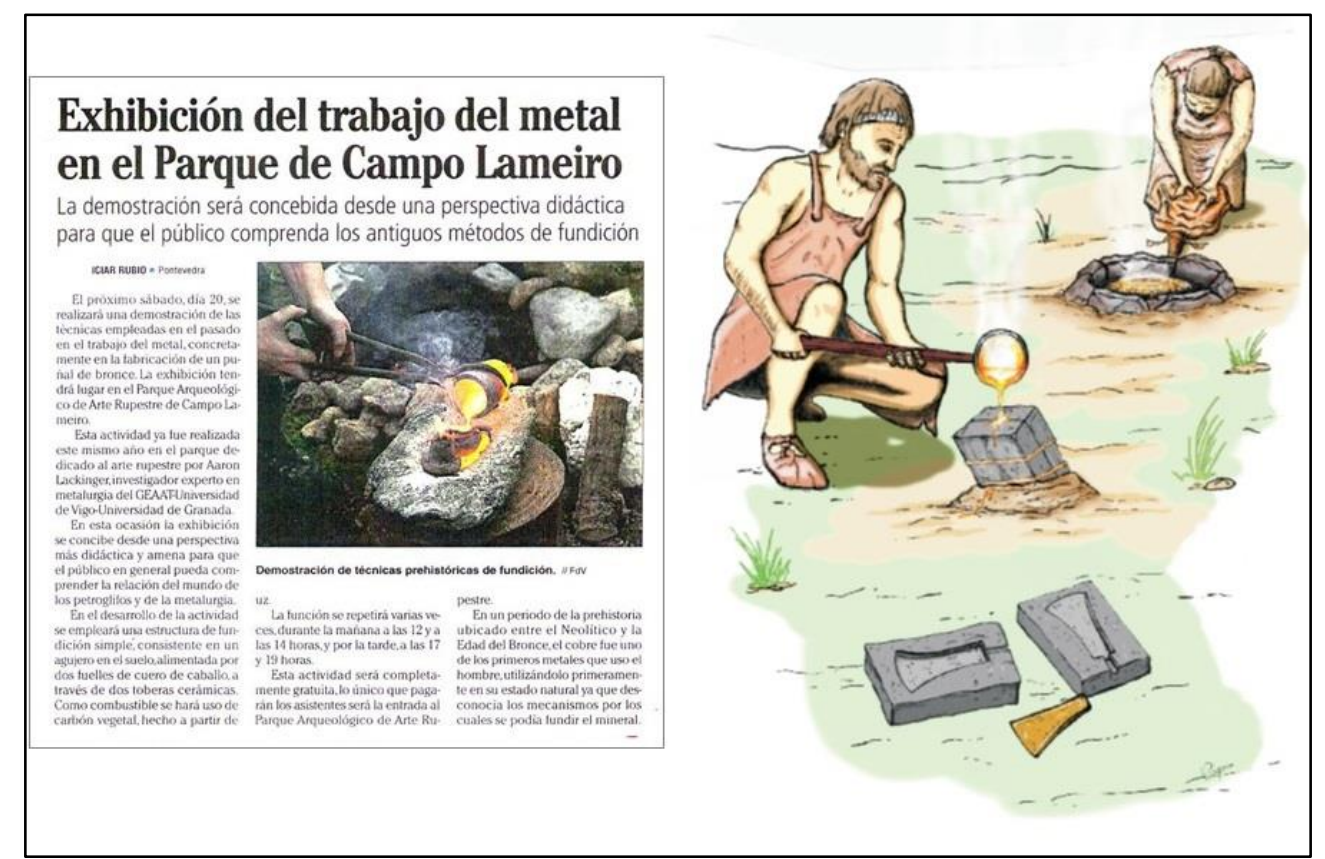

Figura 10. Noticia en prensa de una de las actividades realizadas en el PAAR y boceto de ilustración recreada a partir de estas actividades, de la autoría de Víctor Vázquez Collazo.

Como disciplina que puede unir dos mundos, el del académico especialista, interesado en los estudios analíticos/científicos/arqueológicos y el de la ciudadanía, es fundamental la apuesta por una sensibilización en los medios más académicos y científicos de la importancia de la arqueología experimental y su papel como actividad de transferencia social y educativa, lo que a medio-largo plazo también traerá beneficios para estudios más detallados y científicos del patrimonio, ya en forma de financiación, ya en forma de mayor capacidad de retención de recursos humanos especializados en el patrimonio cultural.

Finalmente permitiría cubrir carencias como la utilización de nuevos lenguajes de comunicación, a pesar de las facilidades tecnológicas; o el desarrollo de materiales específicos para la didáctica.

\section{Conclusiones}


El conjunto de actividades realizadas ha trascendido de un nivel teórico, a uno práctico, experimental, dotándolo de un contenido material, sensorial y mesurable. Esto nos ha permitido profundizar en el conocimiento de la metalurgia prehistórica, tanto a nivel personal, como a nivel social y académico, en distintos ámbitos.

La realización de este tipo de actividades nos permite llevar a cabo un diálogo con distintos ámbitos de la sociedad, en el cual, el aprendizaje es recíproco, ampliándose, con cada experiencia nuestro conocimiento, tanto sobre la, metalurgia como sobre su didáctica "al calor del fuego".

\section{Bibliografía}

ASCHER, R. (1963): "Experimental Archaeology". American Anthropologist, 63: 793816. https://experimentalarchaeology.files.wordpress.com/2011/06/ascherexperimental-archaeology.pdf

BAENA PEYSLER, J. (1997): “Seguimos jugando cuando hablamos de arqueología experimental?". BAEX: Boletín de Arqueología Experimental, 1: 2-5. https://revistas.uam.es/index.php/arqexp/article/view/5809.

BAENA PEYSLER, J., TORRES NAVAS, C. y PALOMO, A. (2019): “¿Seguimos jugando cuando hablamos de arqueología experimental?". BAEX: Boletín de Arqueología Experimental, 13: 1-8. http://dx.doi.org/10.15366/baexuam2018$\underline{19.13 .001}$

BERGER, D., FIGUEIREDO, E., BRUGMANN, G. y PERNICKA, E. (2018): “Tin isotope fractionation during experimental cassiterite smelting and its implication for tracing the tin sources of prehistoric metal artefacts". Journal of Archaeological Science, 92: 73-86 https://doi.org/10.1016/j.jas.2018.02.006

COMENDADOR REY, B., FIGUEIREDO, E. FONTE, J. y MEUNIER, E. (2016): “La primera minería y metalurgia del estaño en la Península Ibérica: aportaciones al estado de la cuestión”. En J. M. MATA-PERELLÓ, M. A. HUNT ORTIZ y E. ORCHE GARCÍA (eds.): Actas del XV Congreso Internacional sobre Patrimonio Geológico y Minero, pp. 21-40. SEDPGYM- Excmo. Ayuntamiento de Logrosán, Logrosán-Cáceres. http://www.sedpgym.es/index.php/18-publicaciones/actascongresos/141-libro-de-actas-del-xv-congreso-internacionacional-sobrepatrimonio-geologico-y-minero-xix-sesion-cientifica-de-la-sedpgym-logrosan$\underline{2014}$

COMENDADOR REY, B. y MÉNDEZ, L. (2007): “¿Patrimonio o chatarra?: Arqueología experimental y metal”. En M. L. RAMOS SÁINZ, J. E. GONZÁLEZ URQUIJO y J. BAENA PREYSLER (eds.): Arqueología Experimental en la Península Ibérica. Investigación, didáctica y patrimonio, pp. 317-328. Asociación Española de Arqueología Experimental, Santander. 
CUNNINGHAM, P., HEEN, J. y PAARDEKOOPER, R. (eds.) (2008): Experiencing Archaeology by Experiment. Proceedings of the Experimental Archaeology Conference, Exeter, 2007, Oxbow Books, Oxford.

FIGUEIREDO, E., LACKINGER, A., COMENDADOR REY, B., SILVA, R. J. C., VEIGA, J. P. y MIRÃO, J. (2017): “An experimental approach for smelting tin ores from Northwestern Iberia”. Materials and Manufacturing Processes, vol. 32, Iss. 7-8: 765-774.

FLUTSCH, L. (1994): Erare humanum est: bugs and bloopers in antiquity: a special exhibition of the Swiss National Museum Zürich from the 26th October, 1994 to the 30th April, 1995, Swiss National Museum, Zurich.

HEEB, J. y OTTAWAY, S. (2014): "Experimental Archaeometallurgy". En B. W. ROBERTS y C. P. THORNTON (eds.): Archaeometallurgy in Global Perspective: Methods and Syntheses, pp. 161-192. Springer Science + Business Media, New York.

LACKINGER, A. (2014): “Una aproximación experimental al empleo de la esteatita en la metalurgia prehistórica". En F. J. GONZÁLEZ de la FUENTE, E. PANIAGUA VARA y P. de INÉS SUTIL (coords.): Investigaciones Arqueológicas en el valle del Duero, del Paleolítico a la Antigüedad Tardía: actas de las III Hornadas de Jóvenes Investigadores del Valle del Duero, Salamanca, 20, 21 y 22 de noviembre de 2013, Vol. 3, pp. 343-357. Glyphos, Valladolid. https://dialnet.unirioja.es/descarga/articulo/5048785.pdf

LACKINGER, A. (2016): La introducción del estaño en la metalurgia prehistórica peninsular: una perspectiva histórica y tecnológica, Trabajo de Fin de Máster Inédito, Universidad de Granada-Universidad de Vigo, Granada.

LACKINGER, A. y COMENDADOR REY, B. (2013): "From Wax to Metal: An Experimental Approach to the Chaîne Opératoire of the Bronze Disk from Urdiñeira". EXARC Journal, 3. http://journal.exarc.net/issue-2013-3/ea/waxmetal-experimental-approach-chaine-operatoire-bronze-disk-urdineira

LACKINGER, A., COMENDADOR REY, B., FIGUEIREDO, E., ARAÚJO, M. F., SILVA, R. y ROVIRA LLORÉNS, S. (2013): "Copper + Tin + People: Public Co-Smelting Experimentation in Northwestern Iberia”. EXARC Journal, 3. http://journal.exarc.net/issue-2013-3/ea/copper-tin-people-public-co-smeltingexperimentation-northwestern-iberia

LACKINGER, A., FERNÁNDEZ FERNÁNDEZ, C. I., COMENDADOR REY, B., FIGUEIREDO, E., VEIGA, J. P. y SILVA, R. J. C. (2017): "Sacar el estaño de las piedras: Un procedimiento artesanal para la obtención de estaño en la Galicia meridional". En L. J. GARCÍA-PULIDO, L. ARBOLEDAS MARTÍNEZ, E. ALARCÓN GARCÍA y F. CONTRERAS CORTÉS (eds.): Presente y futuro de 
los paisajes mineros del pasado: Estudios sobre minería, metalurgia y poblamiento, pp. 259-267. Editorial Universidad de Granada, Granada.

LEROI-GOURHAN, A. (1971): El Gesto y la palabra, Ediciones de la Biblioteca de la Universidad Central de Venezuela.

MARTINÓN-TORRES, M. (2002): "Chaîne Operatoire: the concept and its applications within the study of technology". Gallaecia, 21: 29-43. https://dialnet.unirioja.es/descarga/articulo/265493.pdf

MORGADO RODRÍGUEZ, A. y BAENA PREYSLER, J. (2011): “Experimentación, Arqueología experimental y experiencia del pasado en la Arqueología actual”. En A. MORGADO RODRÍGUEZ, J. BAENA PREYSLER y D. GARCÍA GONZÁLEZ (eds.): La investigación experimental aplicada a la arqueología, pp. 21-28. Universidad de Granada, Granada. https://arqueologiaexperimental.unican.es/wpcontent/uploads/2017/09/CongresoArqRonda.pdf

RAMOS SÁINZ, M. L., GONZÁLEZ URQUIJO, J. E. y BAENA PREYSLER, J. (coords.) (2006): Arqueología Experimental en la Península Ibérica: Investigación, didáctica y patrimonio, Asociación Española de Arqueología Experimental, Santander. https://arqueologiaexperimental.unican.es/wpcontent/uploads/2017/09/CongresoArqExpSantander.pdf

REEVES, J. y PAARDEKOOPER, R. (eds.) (2014): Experiments Past. Histories of Experimental Archaeology, Sidestone Press, Leiden. https://www.sidestone.com/books/experiments-past

ROVIRA LLORÉNS, S. (2007): "La producción de bronces en la Prehistoria". En J. MOLERA i MARIMON, J. FARJAS i SILVA, P. ROURA i GRABULOSA y T. PRADELL i CARA (eds.): Avances en Arqueometría 2005. Actas del VI Congreso Ibérico de Arqueometría, pp. 21-35. Universitat de Girona, Girona. https://www.sapac.es/actas/VI_Congreso_2005.pdf

ROVIRA LLORÉNS, S (2011-12): “Arqueometalurgia experimental en el departamento de Prehistoria y Arqueología de la U.A.M.”. CuPAUAM, 37-38: 105-120. https://repositorio.uam.es/handle/10486/12437 